\title{
A Theoretical Framework of HIV: Sigmoidal Vertical Transmission Function
}

\author{
Moatlhodi Kgosimore and Gosalamang Ricardo Kelatlhegile* \\ Department of Basic Sciences, Botswana University of Agriculture and Natural Resources, Botswana
}

Submission: March 8, 2016; Published: May 12, 2017

*Corresponding author: Gosalamang Ricardo Kelatlhegile, Department of Basic Sciences, Botswana University of Agriculture and Natural Resources, Botswana, Email: gkelatlhegile@gmail.com

Abstract

HIV/AIDS has over three decades caused loss of life among infected individuals and reversed socio-economic gains in both developed and developing nations, particularly in sub-Saharan Africa. The HIV infection is mainly transmitted horizontally and vertically. This study formulates and analyses an HIV theoretical framework described by sigmoidal vertical transmission function. We model the proportion vertically infected with a time dependent sigmoidal function, which is a decreasing function of time. The aim of the study is to investigate the role vertical transmission in predicting the outcome of an epidemic. A system of differential equations based on continuous time is formulated and simulated for various parameter values and state variables at equilibrium.

Keywords: Vertical transmission; Sigmoidal, Prevention; Treatment

\section{Introduction}

Scientific and medical discoveries have made advances in understanding virology, immunology and pathogenesis of HIV infection. These advances have led to the design of effective prevention and treatment strategies of HIV infection in infants, children and adolescents. For instance, antiretroviral therapy (ART) during pregnancy, delivering and breast feeding can eliminate mother-to-child transmission. There are challenges associated with ART implementation especially in resource limited settings [1]. Timely initiation of antiretroviral therapy (ART) in HIV infected individuals has drastically reduced mortality and prolonged their lives [1]. Furthermore, improved survival and reduced HIV transmission risks by HIV infected individuals has led to decline in HIV incidence and increase HIV prevalence [1].

According to [2] an estimated that 34 million people were living with HIV at the end of 2011. Among these, 330000 were children less than 15 years, 2.5 million new HIV infected people. Vertical HIV transmission from mother-to-child accounts for more than $90 \%$ of paediatric AIDS [2-4]. Sub-Saharan Africa continues to be the world's most affected region $[5,6]$.

In order to prevent and control the spread of infectious diseases, mathematicians have embarked upon studies that investigate the role of treatment functions in epidemiological models. Studies of classical models have revealed that treatment functions are important in decreasing the spread of epidemiological diseases. Hampanda [7] applied theoretical frameworks to understand social barriers to prevention of mother-to-child transmission. Chibaya et al. [8] investigated the effect of vertical transmission and evolution of drug resistance on the dynamics of the disease. Baryarama et al. [3] formulated and analysed an HIV/AIDS model with complacency described by an AIDS population dependent function with inverse relation.

It is upon this background that we consider a mathematical model that investigates the role of sigmoidal treatment functions in predicting the burden of vertical transmission. This paper acknowledges various challenges ranging from financial (unaffordable of drugs), organizational (insufficient facilities), (physical) limited access to treatment or resources to social (cultural, decision making processes). The paper is arranged as follows: section (2) describes model formulation, section (3) model analysis; section (4) numerical simulations and concluding remarks are done in section (5).

\section{The mathematical Model}

We consider an epidemic model which incorporates the effects of vertical transmission described by a sigmoidal function. We divide the population into five compartments, namely, Susceptible S, Asymptomatic infectives X, Symptomatic infectives $\mathrm{Y}$, Treated infectives $\mathrm{Z}$ and AIDS A following the WHO stages of HIV infection. The susceptible population is replenished by births or recruitment at the rate $\pi_{0}$ and through 
births of infection-free individuals at the rate $b(1-\omega(t)$ where is the per capita birth rate and is modelled using a piecewise sigmoidal function, $\omega(t)$ given by

$$
\omega(t)= \begin{cases}\omega_{0} & \text { if } 0 \leq t \leq \tau \\ \omega_{0}+\frac{\omega_{1}-\omega_{0}}{1+e^{-\phi(t-\tau)}} & \text { if } t>\tau .\end{cases}
$$

The function $\omega(t)$ is a decreasing function of time, with parameter $\omega_{0}$ denoting the proportion of children born or recruited HIV positive in the absence of treatment (baseline estimated at $40 \%$, and $\omega(t)$ is the proportion of children born or recruited HIV positive in the presence of treatment (estimated at $5 \%$ ), while $\varphi$ is a nonnegative constant. We observe that $\omega(0)=\omega_{0}, \omega(\infty)=\omega_{1}$ and $\omega_{0} \geq \omega_{1}$. The quantity $\tau$ is time of inception of treatment strategy. All compartments are subject to natural death rate $\mu$. The Asymptomatic, symptomatic, treated HIV infectives and AIDS are subjected to disease-induced death rates $\delta_{x}, \delta_{y}, \delta_{z}$ and $\delta_{a}$ in that order. Treatment of asymptomatic infectives (mother-to-child prevention), symptomatic and AIDS are respectively denoted by $\sigma_{x}, \sigma_{y}$ and $\sigma_{a}$. The modification parameters $\theta_{y}(\geq 1) \theta_{z}(\geq 1)$ and $\sigma_{a}$. account for levels of infectiousness for the respective infected individuals, while $v_{x}, v_{y}$ and $v_{z}$ are respectively progression rates of Asymptomatic, symptomatic and treated infectives to AIDS. The parameter $\alpha_{x}$ is the rate at which asymptomatic infectives develop clinical signs. A parameter $\rho$ is the proportion of HIV positive children recruited into the symptomatic compartment.

The above description leads to a system of differential equations

$$
\begin{aligned}
& \frac{d S}{d t}=\pi_{0}+b(1-\omega(t)) I-B S-\mu S, \\
& \frac{d X}{d t}=b \omega(t)(1-\rho) I+B S-\left(\mu+\alpha_{x}+v_{x}+\delta_{x}+\sigma_{x}\right) X, \\
& \frac{d Y}{d t}=b \rho \omega(t) I+\alpha_{x} X-\left(\mu+v_{y}+\delta_{y}+\sigma_{y}\right) Y, \\
& \frac{d Z}{d t}=\sigma_{x} X+\sigma_{y} Y+\sigma_{a} A-\left(\mu+v_{z}+\delta_{z}\right) Z, \\
& \frac{d A}{d t}=v_{x} X+v_{y} Y+v_{z} Z-\left(\mu+\delta_{a}+\sigma_{a}\right) A .
\end{aligned}
$$

with initial conditions $S(0)=S_{0}, X(0)=X_{0}, Y(0)=Y_{0}, Z(0)=Z_{0}, A(0)=A_{0}$ and the total population given by $N=S+X+Y+Z+A$. The force of infection is given by

$$
B=c \beta\left(\frac{X+\theta_{y} Y+\theta_{z} Z+\theta_{a} A}{N}\right)=c \beta\left(\frac{I}{N}\right)
$$

Where

$$
I(t)=X(t)+\theta_{y} Y(t)+\theta_{z} Z(t)+\theta_{a} A(t)
$$

The parameter c measures the effective contacts and is the probability of transmission per contact. Adding all the equations of system (1), we obtain changes in the total population describe by

$$
\begin{aligned}
\frac{d N}{d t} & =\pi_{0}+b I-\mu N-\delta_{x} X-\delta_{y} Y-\delta_{z} Z-\delta_{a} A \\
& \leq \pi_{0}+b I-(\mu+\delta) N
\end{aligned}
$$

where $\delta=\min _{i}\left(\delta_{i}\right)$ for $i=a, x, y, z$. In the absence of infection, integration of the inequality ( 3 ) yields

$$
\limsup _{t \rightarrow \infty} N(t) \leq \frac{\pi_{0}}{\mu+\delta} \leq \frac{\pi_{0}}{\mu} .
$$

Thus, the total population is limited by $N=\frac{\pi_{0}}{\mu+\delta} \leq \frac{\pi_{0}}{\mu}$. The distinction between this model and other models of HIV is that we allow for treatment of AIDS individuals who recover from the disease to treated class. We attempt to solve a non-autonomous system of equations to predict the epidemic outcome based on the cumulative infective cases

$$
C(t)=X(t)+Y(t)+Z(t)+A(t) .
$$

\section{Invariant region}

We analysis and discuss model (1) in a biological feasible region given by

$$
\Omega=\left\{(S, X, Y, Z, A) \in R_{+}^{5}: N \leq \frac{\pi_{0}}{\mu+\delta} \leq \frac{\pi_{0}}{\mu}\right\} .
$$

It is easy to show that the region $\Omega$ is positively invariant and it is sufficient to consider solutions in $\Omega$. Hence, all solution of model (1) starting in $\Omega$ remain in $\Omega$ for all $t \geq 0$. All the parameters and state variables for model (1) are assumed to be nonnegative for $t \geq 0$, since it monitors changes in the human population. Thus, the model is well posed.

\section{Steady States Solution}

To obtain the equilibrium points, we set the right hand side of the model (1) to zero and solve the system of non-linear equations

$$
\begin{aligned}
& \pi_{0}+b(1-\omega(t)) I^{*}-B^{*} S^{*}-\mu S^{*}=0, \\
& b \omega(t)(1-\rho) I^{*}+B^{*} S^{*}-\left(\mu+\alpha_{x}+v_{x}+\delta_{x}+\sigma_{x}\right) X^{*}=0, \\
& b \rho \omega(t) I^{*}+\alpha_{x} X^{*}-\left(\mu+v_{y}+\delta_{y}+\sigma_{y}\right) Y^{*}=0, \\
& \sigma_{x} X^{*}+\sigma_{y} Y^{*}+\sigma_{a} A^{*}-\left(\mu+v_{z}+\delta_{z}\right) Z^{*}=0, \\
& v_{x} X^{*}+v_{y} Y^{*}+v_{z} Z^{*}-\left(\mu+\delta_{a}+\sigma_{a}\right) A^{*}=0 .
\end{aligned}
$$

After some tedious manipulations we obtain interms of $X^{*}$ as $Y^{*}=M_{0} X^{*}, \quad Z^{*}=P_{0} X^{*}+P_{1} Y^{*}=\left(P_{0}+P_{1} M_{0}\right) X^{*}, I^{*}=\left(Q_{0}+Q_{1} M_{0}\right) X^{*}$ and $A^{*}=\left(U_{0}+U_{1} M_{0}\right) X^{*}$, where

$$
\begin{aligned}
& P_{0}=\frac{\sigma_{x} \Phi_{a}+\sigma_{a} v_{x}}{\Phi_{a} \Phi_{z}-\sigma_{a} v_{z}}, P_{1}=\frac{\sigma_{y} \Phi_{a}+\sigma_{a} v_{y}}{\Phi_{a} \Phi_{z}-\sigma_{a} v_{z}}, U_{0}=\frac{v_{x} \Phi_{z}+\sigma_{x} v_{z}}{\Phi_{a} \Phi_{z}-\sigma_{a} v_{z}}, U_{1}=\frac{v_{y} \Phi_{z}+\sigma_{y} v_{z}}{\Phi_{a} \Phi_{z}-\sigma_{a} v_{z}}, \\
& Q_{0}=1+P_{0}\left(\theta_{z} a+\frac{\theta_{a} v_{z}}{\Phi_{a}}\right)+\frac{\theta_{a} v_{z}}{\Phi_{a}}, Q_{1}=\theta_{y}+P_{1}\left(\theta_{z}+\frac{\theta_{a} v_{z}}{\Phi_{a}}\right) \theta_{y}+\frac{\theta_{a} v_{y}}{\Phi_{a}}, \Phi_{a} \Phi_{z}-\sigma_{a} v_{z}>0, \\
& \Phi_{x}=\mu+\alpha_{x}+v_{x}+\delta_{x}+\sigma_{x}, \Phi_{y}=\mu+v_{y}+\delta_{y}+\sigma_{y}, \Phi_{z}=\mu+v_{z}+\delta_{z}, \Phi_{a}=\mu+\delta_{a}+\sigma_{a} \\
& \text { and } M_{0}=\frac{\alpha_{x}+b \rho \omega(t) Q_{0}}{\Phi_{y}-b \rho \omega(t) Q_{1}} .
\end{aligned}
$$

Define the basic reproduction number under vertical transmission or the average number of secondary cases generated by an infective individual during his/her infectious period as 


$$
R_{0}(t)=\eta c \beta, \text { where } \eta=\eta(t)=\frac{Q_{0}+Q_{1} M_{0}}{\Phi_{x}-b \omega(t)(1-\rho)\left(Q_{0}+Q_{1} M_{0}\right)} .
$$

The formula of the reproduction number due to vertical transmission $R_{0}(t)$, allows us to compute the incremental reproduction number $\Delta R_{0}(t)=R_{0}(\infty)-R_{0}(0)=c \beta\left(\eta_{\infty}-\eta_{0}\right)<0$, which measures or assesses the rate of reduction of new infections per unit of time. Knowledge of this quantity may help adjust some of the critical control factors to achieve desired results. Now, combining equations in system (6) and $B^{*}=c \beta\left(\frac{I^{*}}{N^{*}}\right)$, we obtain

$$
X^{*}=0
$$

$$
\begin{aligned}
& \text { or } \\
& R_{0} S^{*}=N^{*},
\end{aligned}
$$

It is worth noting that these solutions hold provided $\Phi_{y}-b \rho \omega(t) Q_{1}>0$ and $\Phi_{x}-b \omega(t)(1-\rho)\left(Q_{0}+Q_{1} M_{0}\right)>0$.

\section{Disease-free equilibrium}

The solution (7) leads to the disease-free equilibrium given by

$$
E_{0}=\left(\frac{\pi_{0}}{\mu}, 0,0,0,0\right)
$$

and it exists for all values of Linearization of system (1) and construction of appropriate Lyapunov function [9] of the form

$$
V=\sum_{i=1}^{4} x_{i}^{*}\left(\frac{x_{i}}{x_{i}^{*}}-\ln \frac{x_{i}}{x_{i}{ }^{*}}\right)
$$

We are able to establish the following theorem:

Theorem 3.1: The disease-free equilibrium (DFE) (exists for all $R_{0}$ ) is locally and globally asymptotically stable $R_{0}<1$.if and unstable if $R_{0}>1$.

\section{Endemic equilibrium}

The result (8) yields the endemic equilibrium whose coordinates can be expressed in terms of $B^{*}$ and $S^{*}$ as

$$
\begin{aligned}
S^{*} & =\frac{\pi_{0}}{\mu+[1-b(1-\omega(t)) \eta] B^{*}}, \quad X^{*}=\frac{\pi_{0} \eta B^{*}}{\mu+[1-b(1-\omega(t)) \eta] B^{*}}=\eta B S^{*}, \\
Y^{*} & =\frac{\pi_{0} \eta M_{0} B^{*}}{\mu+[1-b(1-\omega(t)) \eta] B^{*}}=\eta M_{0} B^{*} S^{*}, \quad Z^{*}=\frac{\left[P_{0}+P_{1} M_{0}\right] \pi_{0} \eta B^{*}}{\mu+[1-b(1-\omega(t)) \eta] B^{*}}=\left[P_{0}+P_{1} M_{0}\right] \eta B^{*} S^{*}, \\
A^{*} & =\frac{v_{x} X^{*}+v_{y} Y^{*}+v_{z} Z^{*}}{\Phi_{a}}=\left(\frac{v_{x}+v_{y} M_{0}+v_{z}\left[P_{0}+P_{1} M_{0}\right]}{\Phi_{a}}\right) \eta B^{*} S^{*} .
\end{aligned}
$$

Noting that $N^{*}$ can be expressed as $N^{*}=S^{*}\left(1+K B^{*}\right)$, where $K=\eta\left(1+M_{0}+\left(P_{0}+P_{1} M_{0}\right) P_{2}\right)$ and $P_{2}=\frac{v_{x}+v_{y}, M_{0}+v_{s}\left(P_{0}+P_{i} M_{0}\right)}{\Phi_{a}}$, and upon substituting for $N^{*}$ in (8), we obtain $B^{*}=\frac{1}{K}\left(R_{0}-1\right)$.

The result of the stability analysis of the endemic equilibrium point can be summarized by the following theorem. Theorem 3.2 The endemic equilibrium (EE) exists and it is locally asymptotically stable if $R_{0}>1$ and unstable if $R_{0}<1$ :

\section{Numerical Simulation}

To illustrate analytical results, we simulated system (1) for various values of treatment parameters $\sigma_{i}$ and time of treatment therapy inception $\tau$ (Figure 1). The results of the study show that increasing treatment rates have the effect of reducing the burden of vertical transmission (Figure 2). Reduction in $\tau$ (early treatment), has the effect of reducing $\omega(t)$ and consequently the proportion of children born HIV positive. The effects of vertical transmission of HIV/AIDS cannot be underestimated because they have serious psychological consequences among children, adolescents and adults who acquired infection via vertical transmission. These consequences may have detrimental effects in the effective implementation of intervention strategies. It is therefore, imperative that mathematical models provide accurate estimates in the timing of inception of therapy (treatment and preventive) as well as prediction of epidemic outcomes of interventions for continual refinement of strategies.

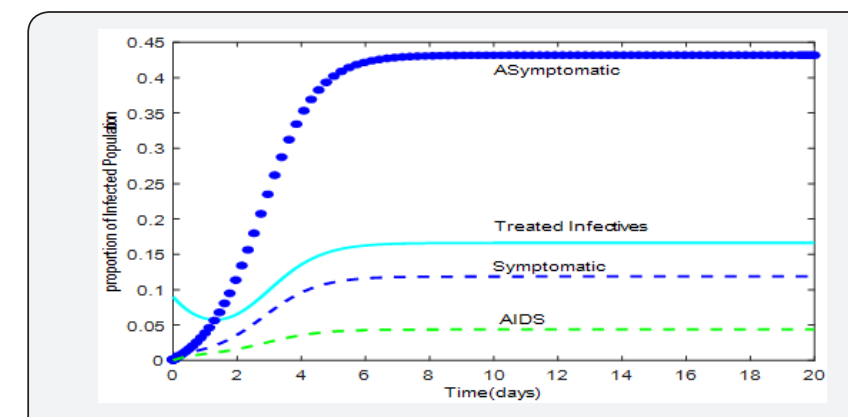

Figure 1: Trajectories of the infected population.

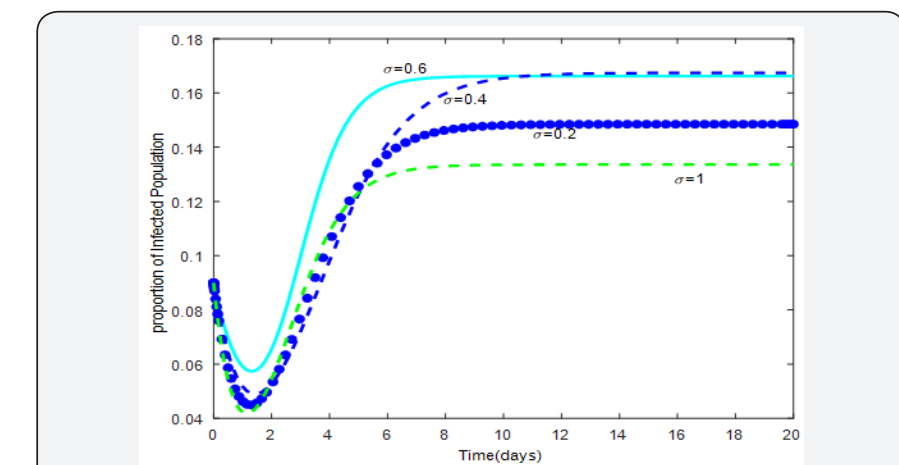

Figure 2: Dynamics of the infected population with variation of treatment rates.

\section{Conclusion}

The study formulated and analyzed an HIV theoretical framework described by sigmoidal vertical transmission function with the aim of investigating the role of vertical transmission in predicting the outcome of an epidemic. The study shows that the disease clears from the population if $R_{0}<1$ and persist if $R_{0}>1$ : Furthermore, the study underscores the importance of estimation of critical factors influencing disease transmission dynamic. We believe our model formulation, presents a useful framework, which can be calibrated with available data to obtain insights in effects of vertical transmission in the presents of treatment therapy. The study may further aid determination of optimal time control for treatment inception to bring about elimination of vertical transmission. 


\section{Acknowledgement}

The researcher is deeply indebted to the Botswana University of Agriculture and Natural Resources (BUAN) for conducive environment for conducting this research. The authors would like to thank the reviewers for their valuable suggestions for the betterment of the article.

\section{References}

1. Mutevedzi PC, Newell ML (2014) The changing face of the HIV epidemic in sub-Saharan Africa, Trop Med Int Health 19(9): 1015-1028.

2. Korobeinikov A, Wake GC (2002) Lyapunov functions and global stability for SIR, SIRS and SIS epidemiological models. Applied Mathematics Letter 15: 955-960.

3. Baryarama F, Mugisha JYT, Luboobi LS (2006) Mathematical model for HIV/AIDS with complacency in a population with declining prevalence. Computational and Mathematics methods in Medicine 7(1): 27-35.
4. Brilie B, Diriba TA, Sisay K, Gurmessa A, Seyoum D, et al. (2016) Motherto-child HIV transmission and its predictors among HIV-exposed infants: A Retrospective follow-up study in Southwest Ethiopia. J AIDS Clin Res 7: 9.

5. UNAIDS (2012) Global HIV/AIDS response: Epidemic update and health sector progress toward universal access: progress (2011) World Health Organization, Geneva, Switzerland, pp. 233.

6. Hampanda K (2013) Vertical transmission of HIV in sub-Saharan Africa: Applying theoretical framework in understanding social barriers to PMTCT, ISRN Infectious diseases 2013: 1-5.

7. Kelatlhegile GR, Kgosimore M (2016) Bifurcation analysis of vertical transmission model with preventive strategy. Journal of the Egyptian Mathematics Society 24(3): 492-498.

8. Chibaya S, Kgosimore M, Massawe ES (2013) Mathematical analysis of drug resistance in vertical transmission of HIV/AIDS. Open Journal of Epidemiology 3(3): 139-148.

9. Global AIDS update: Progress report (2016) UNAIDS.

\section{Your next submission with Juniper Publishers}

will reach you the below assets

- Quality Editorial service

- Swift Peer Review

- Reprints availability

- E-prints Service

- Manuscript Podcast for convenient understanding

- Global attainment for your research

- Manuscript accessibility in different formats ( Pdf, E-pub, Full Text, Audio)

- Unceasing customer service

Track the below URL for one-step submission https://juniperpublishers.com/online-submission.php 